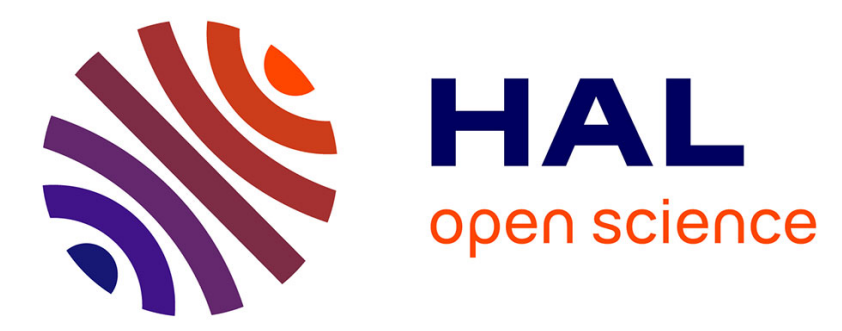

\title{
Potential barrier in the electrostatic sheath around a magnetospheric spacecraft
}

B. Thiébault, A. Hilgers, E. Sasot, H. Laakso, P. Escoubet, Vincent Génot, J. Forest

\section{- To cite this version:}

B. Thiébault, A. Hilgers, E. Sasot, H. Laakso, P. Escoubet, et al.. Potential barrier in the electrostatic sheath around a magnetospheric spacecraft. Journal of Geophysical Research, 2004, 109, pp.12207. 10.1029/2004JA010398 . hal-00290018

\section{HAL Id: hal-00290018 https://hal.science/hal-00290018}

Submitted on 25 Jun 2008

HAL is a multi-disciplinary open access archive for the deposit and dissemination of scientific research documents, whether they are published or not. The documents may come from teaching and research institutions in France or abroad, or from public or private research centers.
L'archive ouverte pluridisciplinaire HAL, est destinée au dépôt et à la diffusion de documents scientifiques de niveau recherche, publiés ou non, émanant des établissements d'enseignement et de recherche français ou étrangers, des laboratoires publics ou privés. 


\section{Potential Barrier in the Electrostatic Sheath Around a Magnetospheric Spacecraft}

B. Thiébault, A. Hilgers, E. Sasot, H. Laakso, P. Escoubet

European Space Agency Keplerlaan 1, 2200 AG Noordwijk, The Netherlands.

V. Génot

CNRS/CESR, Toulouse, France

J. Forest

Institutet För Rymdfysik, Kiruna, Sweden

B. Thiébault, European Space Agency, TOS-EES Keplerlaan 1, 2200 AG Noordwijk, The Netherlands. (Benoit.Thiebault@esa.int) 
Abstract. Plasma and field measurements onboard magnetospheric spacecraft can be influenced by the spacecraft potential and emitted particles such as photo-electrons and secondary electrons. There exist contradictory requirements for on one hand minimising the spacecraft potential and on the other hand minimising perturbations in the sheath and contamination by spacecraft generated electrons. Assessment and mitigation of such effects therefore requires improved quantitative modelling of the spacecraft electrostatic sheath. In this paper a fully self-consistent model of the plasma around an electron emitting central body in a spherically symmetric geometry is used to analyse the electrostatic sheath around an idealised magnetospheric spacecraft. Although the model is too simplistic to allow detailed comparisons with observations it helps to analyse the phenomenon of potential barrier occurrence and some global characteristics which can be relevant to conductive magnetospheric spacecraft like Geotail or Cluster. It is shown that non monotonic potential with negative potential barrier can exist all around a positively charged spacecraft even in the case of realistic illumination pattern. The magnitude of the potential barrier in regions of the magnetosphere with large Debye length plasma is found to be as large as a few volts negative for small positive spacecraft potential, but that it quickly vanishes when the potential is increased by a few volts. Furthermore, the location of the potential barrier is found to be much more variable than previously predicted. 


\section{Introduction}

Electron emission on spacecraft surfaces is an important issue for the operation of plasma detectors in space. Outside the ionosphere, the photo-emission current density has a saturation level typically between 20 and $60 \mu A \cdot m^{-2}$ and may significantly influence the electrostatic potential of spacecraft surfaces. Positive potentials of up to a few tens of volts positive are commonly observed in the magnetosphere which makes the measurements of low energy particles very difficult [Pedersen et al., 1984]. In addition, plasma measurements can be perturbed by electrons emitted from sunlit or particle irradiated surfaces and returning to the detector entrance [Guernsey and Fu, 1970, Hilgers, 1995, Szita et al., 2001, Wrenn and Heikkila, 1973]. For mission preparation and data analysis purpose, the assessment of such effects can be relatively easy when one can neglect the space charge of particles and when the electrostatic potential is a monotonous decreasing function of the distance from the spacecraft surface [cf e.g, Hamelin et al., 2002, Scime et al. 1994]. However, the situation may be more complicated when the space charge induced by ambient and/or emitted electrons is strong enough to significantly affect the sheath structure and possibly even create electrostatic potential barriers [cf. e.g., Guernsey and Fu, 1970, Whipple, 1976, Zhao et al., 1996]. Numerical methods have been developped to self-consistently compute the plasma density and potential around spherical or cylindrical conductors in the most general conditions of plasma [Bernstein and Rabinowitz, 1959; Laframboise, 1966; Chang and Bienkowski, 1970; Parker 1980]. This paper builds on these previous theoretical work to develop a computer model of a sheath dominated by photo-electrons around a sphere. The method is validated by comparison with the 
predictions of a Particle-In-Cell code [Forest et al., 2001]. The method is then applied to the case of Geotail and Cluster low density plasma environments as encountered in the magnetospheric lobes. The results predict a potential barrier which can be of the order of $-1 \mathrm{~V}$ in the low density regions of the magnetosphere and located at a distance from the spacecraft much more variable as a function of the spacecraft potential than predicted earlier by Zhao et al. [1996].

\section{Numerical Model}

In this section the details of the numerical method is given for completeness of the report. It must be noted, however, that regarding the theoretical assumptions, the analytical development and the numerical methods, there is essentially nothing new compared to previously published work which summarised in Parrot et al. [1982]. Therefore, a reader already acquainted with these methods could proceed directly to the next section.

Thanks to the very long mean free path of the plasma particles and the short time scale to reach electrostatic equilibrium compared to change of the environment, collisions between particles and time dependence can be discarded. Furthermore, at the high altitudes relevant to the spacecraft considered in this paper, the magnetic field effects can be neglected. The sheath structure around the electron emitting body is assumed to be well described by the Poisson-Vlasov system of equations with appropriate boundary conditions. The velocity probability density distribution functions of the ambient plasma at large distance from the body (i.e., far from any electrostatic influence of the body) and of the photo-electrons at the body surface are assumed to be Maxwellian. This assumption is supported by the study of Grard [1973] who has shown that for the purpose of average description of the electrostatic environment of spacecraft the speed distribution of photo- 
THIEBAULT ET AL.: ELECTROSTATIC SHEATH AROUND A MAGNETOSPHERIC SPACECRAFT - 5 electrons can be approximated by a Maxwellian distribution. Furthermore, the source of photoelectrons is assumed to be spherically symmetric. A priori, this assumption is more relevant to secondary emission process under irradiation by energetic electrons rather than by UV, which usually comes only from one direction. This aspect is further discussed in Section 3.2. Thanks to the spherical symmetry of the system, the number densities of each population and the potential distribution can be expressed as functions of the sole radial distance from the central body.

\subsection{Poisson Equation}

The electric potential is related to the space charge density by the Poisson equation, which can be written for a problem with spherical symmetry as :

$$
\Delta_{R} \Phi(R)=-\frac{\rho(R)}{\epsilon_{0}}
$$

where $\Delta_{R}$ is the Laplacian in spherical symmetry, $R$ is the radial distance from the central body, and $\Phi(R)$ and $\rho(R)$ are respectively the electric potential and the space charge density at the distance $R$ and $\epsilon_{0}$ is the permittivity of vacuum.

\subsection{Vlasov Equation}

For any particle species $i$, the distribution function $f_{i}$ is related to the number density $N_{i}$ by the equation:

$$
N_{i}(R)=\iint f_{i}\left(R, V_{i}\right) d^{3} V_{i}
$$

where the symbol $V_{i}$ is used for the velocity of species $i$.

In absence of collisions and magnetic field, the distribution function fulfils the Vlasov equation: 


$$
V_{i} . \nabla f_{i}-q_{i} \nabla \Phi . \nabla_{p} f_{i}=0
$$

A consequence of the Vlasov equation is that $f_{i}$ is constant along a particle trajectory, leading to:

$$
f_{i}(R, V)=H_{i}(R, V) \cdot f_{i, S}\left(V_{S}\right)
$$

where the "step-function" $H_{i}$ contains the trajectory information, such that $H_{i}=1$ if the trajectory of the particle at $R$ connects to the source where particles with velocity $V$ exist, and $H_{i}=0$ otherwise. The subscript $S$ is used for the parameters at the source. In this paper, Maxwellian distributions of the density probability of particle velocity at the source are used:

$$
f_{i, S}\left(V_{i, S}\right)=\alpha_{i} N_{i, S}\left(\frac{m_{i}}{2 \pi k_{B} T_{i}}\right)^{\frac{3}{2}} \cdot \exp \left(-\frac{m_{i} V_{i, S}^{2}}{2 k_{B} T_{i}}\right)
$$

where

$$
\alpha_{i}=\left\{\begin{array}{l}
1, \text { for ambient particles } \\
2, \text { for secondary particles }
\end{array}\right.
$$

The $\alpha_{i}$ coefficient is different for the sources of ambient and secondary particles because the normalisation of $f_{i}$ is performed on the whole space for ambient particles and on a half space for the secondary ones (emission from a locally planar surface).

Using the dimensionless quantities:

$$
\begin{gathered}
\epsilon_{i}=\frac{E_{i}}{k_{B} T_{i}} \\
v_{i, S}^{2}=\frac{m_{i} V_{i, S}^{2}}{2 k_{B} T_{i}} \\
\phi_{i, S}=\frac{q_{i} \Phi_{S}}{k_{B} T_{i}}
\end{gathered}
$$


one obtains:

$$
f_{i, S}\left(V_{i, S}\right)=\alpha_{i} N_{i, S}\left(\frac{m_{i}}{2 \pi k_{B} T_{i}}\right)^{\frac{3}{2}} \cdot \exp \left(-v_{i, S}^{2}\right)
$$

The conservation of the total energy implies

$$
\epsilon_{i}=v_{i}^{2}+\phi_{i}=v_{i, S}^{2}+\phi_{i, S}
$$

hence

$$
v_{i, S}^{2}=\epsilon_{i}-\phi_{i, S}
$$

For the ambient particles, the source is at potential zero (i.e., potential at infinity), while for the secondary particles the source is at the spacecraft potential:

$$
\left\{\begin{array}{l}
\phi_{i, S}=\phi_{\infty}=0, \text { for ambient particles } \\
\phi_{i, S}=\phi_{S C}, \text { for secondary particles }
\end{array}\right.
$$

Where $\phi_{S C}$ is the potential of the central body.

Equations (4), (5) and (6) lead to:

$$
f_{i}(R, V)=\alpha_{i} \cdot N_{i, S} \cdot H_{i}\left(R, V_{i}\right)\left(\frac{m_{i}}{2 \pi k_{B} T_{i}}\right)^{\frac{3}{2}} \cdot \exp \left(-\epsilon_{i}+\phi_{i, S}\right)
$$

\subsection{Number Density Computation}

\subsubsection{Integral expression}

Using dimensionless quantities:

$$
n_{i}(r)=\frac{N_{i}(r)}{N_{i, S}}
$$

one obtains:

$$
n_{i}(r)=\frac{\alpha_{i} \cdot \exp \left(\phi_{i, S}\right)}{\pi^{3 / 2}} \iint H_{i}\left(r, v_{i}\right) \cdot \exp \left(-\epsilon_{i}\right) d^{3} v_{i}
$$


Using spherical coordinates,

$$
d^{3} v_{i}=v_{i}^{2} d v_{i} d \psi d(\cos \theta)
$$

where $\theta$ and $\psi$ are respectively the inclination and the azimuth of the velocity vector with respect to a reference axis, one obtains the same Equation as Parrot et al. [1982]

$$
\begin{aligned}
n_{i}(r)= & \frac{2 \alpha_{i} \cdot \exp \left(\phi_{i, S}\right)}{\sqrt{\pi}} \int_{0}^{\infty} v_{i}^{2} \exp \left(-\epsilon_{i}\right) \\
& \times \int_{\theta=0}^{\theta=\pi} H_{i}\left(r, v_{i}\right) \cdot d(\cos \theta) d v_{i}
\end{aligned}
$$

after a trivial integration over $\psi$.

Let $l_{i}$ be the dimensionless angular momentum of the considered type of particle:

$$
\begin{gathered}
l_{i}=\frac{L}{\sqrt{2 m_{i} k_{B} T_{i} R_{S C}}} \\
l_{i}^{2}=r^{2} v_{i}^{2} \sin ^{2} \theta \\
\cos \theta=\sqrt{1-\frac{l_{i}^{2}}{r^{2} v_{i}^{2}}} \\
\int_{\theta=0}^{\theta=\pi} H_{i}\left(r, v_{i}\right) \cdot d(\cos \theta)=\sum_{j} \xi_{j}\left[\frac{1}{v_{i} r} \sqrt{v_{i}^{2} r^{2}-l_{i}^{2}}\right]_{\left(l_{i}^{2}\right)_{\text {min }}}^{\left(l_{i}^{2}\right)_{\text {max }_{j}}}
\end{gathered}
$$

where $\xi_{j}= \pm 1$ and $R_{S C}$ is the radius of the central body. $\left(l_{i}^{2}\right)_{\min _{j}}$ and $\left(l_{i}^{2}\right)_{\max _{j}}$ take into account the $H_{i}$ factor as described previously by the orbits analysis.

When changing the variable $v_{i}$ to $\epsilon_{i}$ as defined above, Equation (8) leads to

$$
\begin{aligned}
n_{i}(r)= & \frac{2 \alpha_{i} \cdot \exp \left(\phi_{i, S}\right)}{\sqrt{\pi}} \int_{0}^{\infty} \exp \left(-\epsilon_{i}\right) \\
& \times \sum_{j} \xi_{j}\left[\frac{1}{2 r} \sqrt{r^{2}\left(\epsilon_{i}-\phi_{i}\right)-l_{i}^{2}}\right]_{\left(l_{i}^{2}\right)_{\text {min }_{j}}}^{\left(l_{i}^{2}\right)_{\text {max }_{j}}} d \epsilon_{i}
\end{aligned}
$$


which can be further simplified to

$$
n_{i}(r)=\frac{2 \alpha_{i} \cdot \exp \left(\phi_{i, S}\right)}{\sqrt{\pi}} \int_{0}^{\infty} \exp \left(-\epsilon_{i}\right) M_{n_{i}}\left(\epsilon_{i}\right) d \epsilon_{i}
$$

where

$$
M_{n_{i}}\left(\epsilon_{i}\right)=\sum_{j} \xi_{j}\left[K_{n_{i}}\left(l_{i}^{2}\right)\right]_{\left(l_{i}^{2}\right)_{\min j}}^{\left(l_{\text {max }}^{2}\right)_{\text {max }}}
$$

with

$$
K_{n_{i}}\left(l_{i}^{2}\right)=\frac{1}{2 r} \sqrt{g_{i}(r)-l_{i}^{2}}
$$

and

$$
g_{i}(r)=r^{2}\left(\epsilon_{i}-\phi_{i}\right)
$$

\subsubsection{Turning point formulation}

The turning point method [cf e.g. Parrot et al. 1982], is based on the analysis of the possible particle orbits to simplify and solve the Vlasov-Poisson system of equations with spherical symmetry. It relies on the identification of the domains where the $H_{i}\left(r, v_{i}\right)$ function is non null.

To illustrate this method, let us consider for an attracted ambient species a very general profile of the function $g_{i}$ as sketched on Figure 1. Let $l_{A}^{2}$ be the absolute minimum of $\mathrm{g}$ over all radius values, and $l_{B}^{2}$ be the absolute minimum of $g_{i}$ for radii $\geq r_{o b s}$, where $r_{o b s}$ is the observation point. In this example, the computation of the density of ambient particles at the location $r_{\text {obs }}$ requires to distinguish 4 cases for particles with a given energy as follows:

- case 1: these particles have a high enough energy to go from the source (i.e. infinity in this example) to the central body surface. 
- case 2: the particles encounter their turning point before reaching the satellite surface (at $\left.r\left(l_{A}^{2}\right)\right)$. Thus, at the observation point, these particles are seen twice for the number density computation.

- case 3: the particles encounter a turning point before reaching the central body surface (at $\left.r\left(l_{B}^{2}\right)\right)$ and return to the ambient plasma. These particles are not seen at the point of observation and therefore not counted in the computation of the number density at this point.

- case 4: this corresponds to particles which are in principle forbidden to reach this region because there is no trajectory connecting them to infinity. Therefore, these particles are neglected.

In this example one eventually obtains:

$$
M_{n_{i}}\left(\epsilon_{i}\right)=K_{n_{i}}(0)+K_{n_{i}}\left(l_{A}^{2}\right)-2 K_{n_{i}}\left(l_{B}^{2}\right)
$$

\subsubsection{Computer code}

The method above can be easily made automatic via a computer scheme. For a given radius $r$, the procedure reduces to finding local minima of the turning point function $g_{i}(r) \equiv r^{2}\left(\epsilon_{i}-\phi_{i}(r)\right)$ defined earlier and to integrate over the authorised values of $l_{i}^{2}$. The density of each species can then be determined by an integration over the energy. Parrot et al. [1982] made use of a relatively low order quadrature method of the energy integral. We found that such a method is not stable for large Debye length values and therefore we made use of a classic Riemann integration method (cf. Appendix A). Once the density of each species is known one can compute the total electric charge and inject it in the Poisson equation, which is solved via a standard numerical scheme, i.e., Gauss-Seidel in 
THIEBAULT ET AL.: ELECTROSTATIC SHEATH AROUND A MAGNETOSPHERIC SPACECRAXT- 11 one dimension. The solution to the Poisson-Vlasov system is then found iteratively using a classic under-relaxation method.

\section{Code Validation}

To validate this method, two tests were performed, which are described below. The first one is based on the comparison with Laframboise [1966] numerical results for a central body in a Maxwellian plasma at equilibrium without photo-emission. The second test includes photoelectrons and comparisons are made with the predictions of a PIC simulation code, PicUp3D, developed by Forest et al. [2001].

\subsection{Comparison with Laframboise's Model}

Laframboise [1966] developed a model of the sheath structure for a Maxwellian plasma around a central body without photo-emission. In this model the infinite plasma is replaced by an outer boundary at finite radius beyond which a power-law potential is specified. The potential profile corresponding to the plasma conditions of Table 1 and shown in Figure 2 of this paper has been derived from Figure 13 of the report by Laframboise [1966].

It must be noted that although such values of the plasma parameters correspond to a plasmaspheric regime the value of the spacecraft potential is chosen to strictly reproduce the regime of parameters of Laframboise's report for test purpose and is certainly not intended to correspond to any realistic operational regime for the spacecraft potential. The potential profile corresponding to vacuum conditions is also shown on Figure 2. The significance of the space charge effects in this regime can be determined by the departure of Laframboise curve from this inverse law. 
It can be seen that the potential profile predicted by the code developed in this paper, also shown on Figure 2 matches very closely the model of Laframboise [1966].

\subsection{Comparison with PicUp3D}

The PicUp3D code is a simulation software dedicated to model spacecraft plasma interactions [Forest et al. 2001]. It is an open source software written in Java, freely available on the web. It is based on a 3D Particle-In-Cell (PIC) kinetic description of ions and electrons to simulate plasma dynamics. Several simulations have been performed for a Maxwellian plasma environment described in Table 2. This plasma corresponds to a regime of parameters for which PicUp3D can provide very stable and accurate results (less than $5 \%$ error on the potential profile) for a computation box of reasonable size, thanks to the ratio of the central body radius over the Debye length close to unity. Again, it must be noted that this regime is used for the purpose of model testing only and is not intended to represent any realistic spacecraft potential operation mode. The potential profiles predicted with PicUp3D are shown on Figure 3 together with the profile computed with the turning point formulation based method. It can be seen that the potential profiles computed with the two different methods are in very good agreement and show the occurrence of a negative potential barrier for the electrons coming from infinity. This result can be considered as a successful cross-validation test of both methods especially with regards to their ability to simulate photo-electron expansion around a spherical body and prediction of non monotoneous potential. In the following the term barrier of potential will be used to describe the value of the difference between the potential at infinity and the potential of the minimum of the barrier when it occurs. 


\subsection{Spherically Symmetric Versus Axi-symmetric Emission}

In the code based on the turning point method like in all previous studies referred to in this paper the photo-electron emission is modelled with spherical symmetry. However, since photoelectrons are only emitted on sunlit surfaces and with a rate proportional to the incident flux of the light a realistic photoemission model should not follow a spherical symmetry. To study the influence of the asymmetric emission, simulations have been performed with PicUp3D on a spherical central body illuminated from one direction for the parameters given in Table 2 in the case of $\Phi_{S C}=1 \mathrm{~V}$. The corresponding potential profiles are shown on Figure 4 as a function of the distance along three directions of space: sunward, antisunward and perpendicular to the sun direction. In addition the potential profile obtained for a spherically symmetric emission corresponding to the same value of the total photo-electron current has been calculated by the turning point method. It can be seen that for this regime of parameters, potential barriers are predicted with all methods and along all the directions investigated. The barrier of potential is the deepest on the sunlit side and the lowest on the side opposite to sunlight. It must also be noted that the potential profile obtained under the spherical hypothesis provides a reasonably good approximation of the potential profile along an axis perpendicular to the sun direction obtained with the axi-symmetric emission (for an equal total photoelectron current).

\section{Applications}

In the following, the possibility of the occurrence of potential barriers around spherical equipotential bodies is investigated in the low density magnetospheric lobe regions. In these regions the plasma temperature is of the order of $100 \mathrm{eV}$ and the density is below 1 
particle per cubic centimetre. As a consequence the Debye length is very large and accurate simulation with a PIC code is extremely demanding in term of computer resources. Therefore, the code based on the turning point method is more convenient to use. It must be noted that although the tests have been performed for Debye length of the order of the central body radius, it can be expected that the model is still valid for much longer Debye length, since the hypotheses of the model are not depending on any conditions on the Debye length value.

\subsection{Geotail barrier estimates revisited}

The Geotail mission is a Japanese-US spacecraft designed to operate in the distant geomagnetic tail. It is equipped with an ion emitter to actively control the spacecraft potential [Schmidt et al. 1995]. To assess the occurrence and value of the potential barrier around the spacecraft, Zhao et al [1996] have used the current balance equation derived from simplified formulas of the current density resulting from Whipple [1976]:

$$
\begin{gathered}
J_{p h o}(1+\Delta u) \exp (-\Delta u) \frac{S}{4 \pi R_{S C}^{2}} \\
=J_{e o}\left(1+\frac{T_{p h}}{T_{e}} \Delta u\right) \cdot \exp \left(-\frac{T_{p h}}{T_{e}} \Delta u\right) \cdot \exp \left(-\frac{T_{p h}}{T_{e}} u_{s}\right)+\frac{I}{4 \pi R_{S C}^{2}}
\end{gathered}
$$

where $J_{p h o}$ is the photoelectron saturation current density, $J_{e o}$ the ambient thermal electron current density. $T_{p h}, T_{e}, I, S$ and $R_{S C}$ are respectively the photoelectron and the plasma electron temperature, the total ion current from an ion emitter, the effective photoelectron emissive surface and the radius of the spacecraft. The symbol $u_{s}$ stands for the dimensionless potential of the spacecraft defined by the relation:

$$
u_{s}=\frac{-e \Phi_{S C}}{k_{B} T_{p h}}
$$


THIEBAULT ET AL.: ELECTROSTATIC SHEATH AROUND A MAGNETOSPHERIC SPACECRAXT- 15

$u_{B}$ is the dimensionless potential of the minimum of the barrier and $\Delta u$ is defined by $\Delta u=u_{B}-u_{s}$

It must be noted that while the non-symmetry of the illumination pattern is taken into account in the value of $S$, equation (13) is in principle only valid under the hypothesis of spherical symmetry of the fields and particles.

The corresponding potential barrier value computed numerically by Zhao et al. [1996] is shown on Figure 5. To determine the location of the minimum of the barrier, Zhao et al. [1996] assumed a simple potential profile based on three parameters that were determined by fitting the boundary conditions.

The corresponding value of the location of the minimum of the barrier is plotted on Figure 6.

The turning point formulation method was used to assess the potential barrier and the location of its minimum for the same parameters as used by Zhao et al. (Table 3). The corresponding values are plotted on Figures 5 and 6 .

It can be seen from Figure 5 that there is a small discrepancy between the predictions of the barrier value from this paper and the estimates from Zhao et al. [1996]. This discrepancy could be due to the fact that the expression of the current density of the ambient and photo-electrons are approximations valid in the limit of infinite Debye length. Another possible explanation is that the potential of the spacecraft is measured by a method relying on the Geotail Langmuir probe and there is an offset which was assumed to be equal to $+2 \mathrm{~V}$ but on which an uncertainty of a few tenth of Volts is likely to exist. Therefore, it can be concluded that the two methods provide results consistent with each other given the uncertainty attached to the one of Zhao et al. [1996]. 
However, there are very strong discrepancies between the prediction of the location of the minimum of the barrier. As acknowledged by Zhao et al. [1996], their potential barrier position estimate was somehow depending on the arbitrary potential profile, which was chosen. Our results, which are not hampered by such a constraint, provide a new look at the expected behaviour of the barrier. One can see that the distance between the spacecraft wall and the location of the minimum of the potential barrier which was nearly constant in the study of Zhao et al. [1996] is indeed very variable. This distance ranges from a few meters to several tens of meters while the potential changes by only a few Volts.

\subsection{Application to Cluster}

The European Space Agency Cluster mission is constituted of 4 spacecraft on a high inclination elliptical orbit (perigee: 19,000 km, apogee: 119,000 km) designed to explore magnetospheric and solar wind interaction processes. It is equipped with an ion emitter, similar to the Geotail one, to actively control the spacecraft potential in the magnetospheric lobe regions. In these regions the ion emitter is routinely operated with a current of a few tens of $\mu \mathrm{A}$ and the spacecraft voltage falls in the range of a few Volts (compared to a few tens of Volts if the ion emitter were switched off). The spacecraft hub has a cylindrical shape with height 1.3 meter and radius 1.45 meter. To investigate the expected potential barrier characteristics, simulations have been performed for a conductive sphere with a surface equal to the one of the cylinder and hence with a radius of 1.41 meter. The characteristics of the model used are given in Table 4 . It must be noted that the barrier characteristics are given for a spacecraft potential as low as $0 \mathrm{~V}$ although this lower bound is likely to be beyond the capability of the ion emitter in this type of plasma environment. 
The plot of the predicted values of the potential barrier and the location of its minimum is shown on Figures 7 and 8 as a function of the spacecraft potential. It can be seen that the value of the potential barrier can be as low as $-2 \mathrm{~V}$ when the spacecraft potential is null and is decreasing rather rapidly toward zero for increasing value of the spacecraft potential. It is predicted that no more potential barriers exist for a spacecraft potential above $7 \mathrm{~V}$ under the simulation hypotheses. Also it can be noted that the potential barrier minimum location varies very much for a small variation of the spacecraft potential.

To investigate the influence of the ambient plasma electron temperature computations were performed using a temperature of $1 \mathrm{eV}$. The corresponding curves are displayed in Figure 9 and 10. The behaviour is very similar as the one in higher temperature environment but with values of the height of the barrier of potential typically 1 Volt lower.

\section{Discussion}

The quantitative estimates above predict that in magnetospheric regions with density around $1 \mathrm{~cm}^{-3}$ and temperature around $100 \mathrm{eV}$ a spacecraft with a typical dimension of the order of 1 meter may be surrounded by a potential barrier of the order of $1 \mathrm{~V}$ due to photo-emitted electrons if the spacecraft potential is lowered down to a few Volts by, e.g., an active ion emitter. There exists a finite value of the potential beyond which no more barrier appears. The value of this limit in the idealised but non-realistic case of spherical symmetry has been found to be at about $+7 \mathrm{~V}$ in a typical magnetospheric lobe environment. Furthermore, it has been shown that the potential barrier minimum location moves from very close to the spacecraft for very low spacecraft potential to very 
large values (more than 50 satellite radii) for higher value of the potential (but also lower value of the potential barrier height).

Occurrence of potential barriers of the order of $1 \mathrm{~V}$ may be a source of interference with onboard plasma measurements in several respects. First, it prevents the lowest energy electrons to reach detectors located on the spacecraft. Second, it contributes to turn back more photo-electrons to the spacecraft walls hereby modifying the expected spacecraft potential and enhancing contamination of detectors by these electrons. Third, it creates anisotropic potential disturbances which can be felt up to a few tens of meters from the spacecraft hub and can therefore induce artefacts on the electrostatic probes mounted on wire booms used for electric field measurements. Although, the model presented in this paper may usefully contribute to raise awareness of these potential problems and provide indications of when they are likely to occur, there is still a certain number of limitations which have to be addressed before an accurate quantitative modelling would be possible. These limitations are discussed in the following.

The first limitation is the hypothesis of spherical symmetry under which the above results are valid. It is obvious, that even in the case of a roughly spherical spacecraft, the source of photo-electrons itself is strongly non-symmetric. However, thanks to the comparison with a fully 3D model available for lower values of the Debye length there is good confidence that at least the order of magnitude of these estimates are relevant to the value of potential barriers along directions perpendicular to the sun direction axis. Much higher (respectively slightly lower) potential barrier height is expected in the direction (respectively opposite) of the sun. The spherical symmetry is also strongly broken for spacecraft equipped with long wire antennas e.g. for the measurements of magnetospheric 
THIEBAULT ET AL:: ELECTROSTATIC SHEATH AROUND A MAGNETOSPHERIC SPACECRAX̃T- 19 electric field. One can expect that such antennas usually grounded to the hub will affect the sheath by the direct perturbation of potential that they introduce but also by their influence on the photo-electron propagation. In the case of Cluster, it is not clear yet how much these effects will influence the above predictions.

A second limitation is the fact that the device used for lowering the potential may itself strongly affect the sheath characteristics. It can be expected that the Cluser and Geotail plume from the ion emitter can modify the potential and particle density in the sheath. It could on one hand induce a strong positive space charge which may raise the potential over a distance which has to be determined and on the other hand attract a certain amount of photo-electrons. The net results is likely to be a reduction of the potential barrier height (at least at long enough distance from the spacecraft).

Furthermore, it can be noted that actual estimate of the amount of photo-electrons turned back to a detector more critically depends of geometric factor and of details of the photo-electron emission function which are not included in the model presented in this study.

\section{Conclusions}

In this paper, previously established theoretical and numerical methods have been used to develop a code which provides a computer model of the electrostatic sheath around a central electron emitting body in spherical symmetry. The study has confirmed that non-monotonic potential profile with negative potential barrier may be found in a large range of plasma and body parameters including a regime relevant to magnetospheric spacecraft in the lobes, i.e., for a central body with positive potential in a plasma with Debye length of the order of the central body radius or more. Furthermore, it has been 
shown that a barrier of potential may still exist all around the central body even for a more realistic illumination pattern inducing photo-emission only on one side of the central body. In this case, the resulting potential profile in directions perpendicular to the sun direction was shown to be reasonably well approximated with a spherical symmetric model corresponding to an equal total photo-electron current. Applying the code to conditions relevant to the lowest density magnetospheric regions indicated that the potential barrier can be of the order of $1 \mathrm{~V}$ when the spacecraft potential is kept at small potential values (say $\leq 3 \mathrm{~V}$ ) and rapidly decreases to a few tenth of Volts for potential above $5 \mathrm{~V}$.

While it is obvious that the use of techniques to reduce the spacecraft potential in a very low density magnetospheric regions has potentially very strong benefits in improving low particle measurements in general, our results suggest that it is in principle possible to determine a best compromise for spacecraft potential control between the requirement for lowering the spacecraft absolute potential and the one for minimising the occurrence and magnitude of a negative electrostatic potential barrier.

Our quantitative predictions of barrier height are consistent with the potential barrier values estimated with a model based on a simplified current balance equation [Zhao et al., 1996]. Our results extend previous works in two ways. First, they clarify the relevancy of spherically symmetric models to extrapolate to the more realistic non spherically symmetric case. Second, they provide a self-consistent location of the peak of the barrier of potential which has been found to be much more variable than predicted by the previous attempt of Zhao et al. [1996]. Future work to extend the applicability of the code to quantitative modelling of the actual electrostatic sheath of a magnetospheric spacecraft like Cluster must address the influence of the ion plume space charge which may attract a 
THIEBAULT ET AL.: ELECTROSTATIC SHEATH AROUND A MAGNETOSPHERIC SPACECRAXXT- 21 significant amount of photo-electrons and therefore confine the barrier closer to the plume and the long wire boom antennas which considerably complicate the spacecraft and the sheath geometry. Also, in regions where the average plasma thermal velocity is lower than the spacecraft speed relative to the plasma, wake effects will have to be taken into account. These aspects are currently under investigations. Finally, to interpret features of photo-electrons turned back to the detectors [Szita et al. 2001, Fazakerley private communication 2003] a more detailed description of spacecraft geometry and photo-electron distribution is required.

\section{Appendix A: Integration Method}

The integral over the energy (Equation 10) can be performed by an appropriate numerical method, e.g. energy quadrature, as proposed by Parrot et al. [1982], or Riemann integration.

Quadratic formulas have been developped by [Steen et al., 1969] and by [Laframboise and Stauffer, 1969] to evaluate the integrals over energy for the number density computation. The general expression of this approximation is:

$$
\int_{0}^{\infty} \exp \left(-\epsilon_{i}\right) M_{n_{i}}\left(\epsilon_{i}\right) d \epsilon_{i}=\sum_{d=1}^{D} C_{d} M_{n_{i}}\left(\epsilon_{d}\right)
$$

where there are $D$ coefficients $C_{d}$, and $D$ energies $\epsilon_{d}$ with $d=1,2, \ldots D$, which depends on the order $D$ of the quadratic scheme chosen. Let consider $a_{d}$ and $H_{d}$ be respectively the abscissae and the coefficients computed by [Steen et al., 1969]. $C_{d}$ is then defined by: 


$$
C_{d}=2 H_{d} a_{d}
$$

and $\epsilon_{d}$ by:

$$
\epsilon_{d}=a_{d}^{2}
$$

One injects these coefficients in Equation A1 to obtain the integral approximation.

An example of the variation of the predicted barrier level with the level, $D$, is shown on Figure 11 corresponding to the sheath profile computed in section 3.2. It can be seen that in this case the convergence is rapidly achieved.

However, we found that for certain regimes of parameters, the quadratic expression of the integral over energy is strongly oscillating, which results on uncertainties for the potential barrier value (Figure 12) and even more on the location (Figure 13). Figures 12 and 13 show the dependency of the values and positions of the potential barrier in function of the level $D$ of the scheme.

An integration method based on the approximation by a sum of rectangular surfaces has been used instead of the energy quadrature to minimise the uncertainties in the integral computations. This resulted in much more time consuming computations but this method allowed a monotoneous convergence and therefore to reach an accuracy as high as desirable. 
THIEBAULT ET AL.: ELECTROSTATIC SHEATH AROUND A MAGNETOSPHERIC SPACECRAX̄T- 23

\section{Notation}

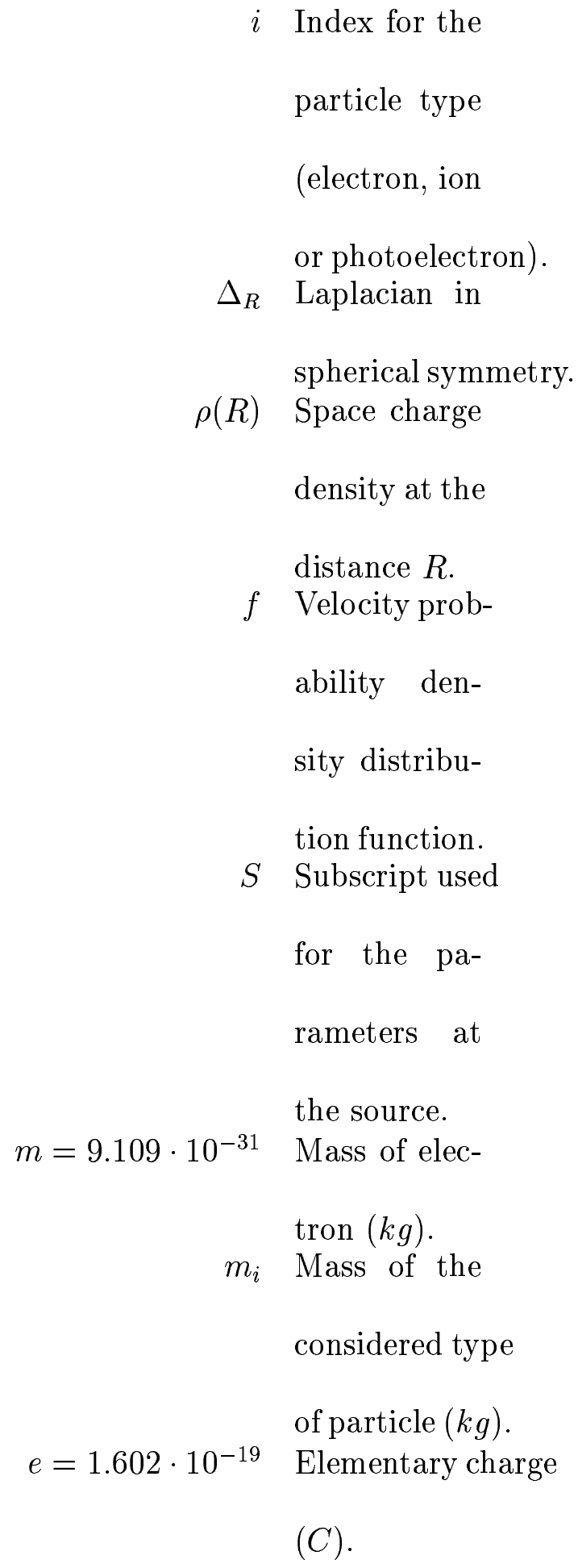




$$
\begin{aligned}
& q_{i}= \pm e \quad \text { Charge of the } \\
& \text { considered pop- } \\
& \text { ulation }(C) \text {. } \\
& k_{B}=8.617 \cdot 10^{-5} \quad \text { Boltzmann con- } \\
& \text { stant }\left(e V \cdot K^{-1}\right. \\
& \epsilon_{0}=8.854 \cdot 10^{-12} \text { Vacuum elec- } \\
& \text { trical permit- } \\
& \text { tivity }\left(F . m^{-1}\right) \text {. } \\
& \Lambda_{D}=\sqrt{\frac{\epsilon_{0} k_{B} T_{i}}{n_{i} e^{2}}} \quad \text { Debye length } \\
& (m) \text {. } \\
& T_{i} \text { Temperature } \\
& (\mathrm{eV}) \text {. } \\
& R \text { Radial distance } \\
& \text { from the cen- } \\
& \text { tre of the cen- } \\
& \text { tral body }(m) \text {. } \\
& R_{S C} \text { central body } \\
& \text { radius }(m) \text {. } \\
& \Phi(R) \text { Potential at } \\
& R(V) \text {. } \\
& \Phi_{S C} \text { Potential at } \\
& \text { probe surface }
\end{aligned}
$$


THIEBAULT ET AL.: ELECTROSTATIC SHEATH AROUND A MAGNETOSPHERIC SPACECRAX̄TL- 25

$$
\begin{aligned}
& N_{i}(R) \text { Number den- } \\
& \text { sity of the } \\
& \text { considered pop- } \\
& \text { ulation at } R \\
& \left(m^{-3}\right) \text {. } \\
& N_{i, S} \text { Number den- } \\
& \text { sity of the } \\
& \text { source for the } \\
& \text { considered pop- } \\
& \text { ulation }\left(m^{-3}\right) \text {. } \\
& V_{i}=\sqrt{\frac{2 k_{B} T_{i}}{m_{i}}} \text { Velocity norm } \\
& \text { of the consid- } \\
& \text { ered particle } \\
& \left(m \cdot s^{-1}\right) . \\
& \theta \text { Angle between } \\
& \text { the velocity } \\
& \text { and the ra- } \\
& \text { dial direction } \\
& \text { (radian). } \\
& E_{i}=\frac{1}{2} m_{i} V_{i}^{2}+q_{i} \Phi \text { Total energy } \\
& (J) \text {. } \\
& L=m_{i} R V_{i} \text { Angular mo- } \\
& \text { mentum }\left(k g \cdot m^{2} \cdot s^{-1}\right) \text {. } \\
& \lambda_{D}=\frac{\Lambda_{D}}{R_{S C}} \text { Dimensionless } \\
& \text { Debye length. }
\end{aligned}
$$




$$
\begin{aligned}
& r=\frac{R}{R_{S C}} \text { Radial distance } \\
& \text { from probe } \\
& \text { centre. } \\
& n_{i}(r)=\frac{N_{i}}{N_{i, S}} \text { Number den- } \\
& \text { sity of the } \\
& \text { considered pop- } \\
& \text { ulation at } r \text {. } \\
& v_{i}=V_{i} \sqrt{\frac{m_{i}}{2 k_{B} T_{i}}} \text { Dimensionless } \\
& \text { velocity norm } \\
& \text { of the consid- } \\
& \text { ered particle. } \\
& \phi_{i}(r)=\frac{q_{i} \Phi(R)}{k_{B} T_{i}} \text { Dimensionless } \\
& \text { potential at } \\
& r \text {. } \\
& \epsilon_{i}=\frac{E_{i}}{k_{B} T_{i}}=v_{i}^{2}+\phi_{i} \text { Dimensionless } \\
& \text { total energy } \\
& \text { of the consid- } \\
& \text { ered type of } \\
& \text { particle. } \\
& l_{i}=\frac{L}{R_{S C} \sqrt{2 m_{i} k_{B} T_{i}}}=r v_{i} \sin \theta \text { Angular momentum }
\end{aligned}
$$

\section{Acknowledgments.}

This study has been performed in the frame of the Spacecraft Plasma Interaction Network in Europe (SPINE) activities (cf. www.spis.org). We acknowledge useful discussions with the participants of the 5th SPINE workshop organized by J.-F. Roussel. We are 
THIEBAULT ET AL.: ELECTROSTATIC SHEATH AROUND A MAGNETOSPHERIC SPACECRAX̄T- 27

greatful to Andrew Fazakerley (Cluster PEACE PI) and to David Cooke for stimulating discussions on the topic and to the two referees for useful comments.

\section{References}

Bernstein, I. B., and I. Rabinowitz, Theory of electrostatic probes in a low-density plasma, Phys. Fluids, Vol. 13, 902, 1970.

Chang, K. W., and G. Bienkowski, Effects of electron emission at arbitrary pressures, Phys. Fluids, Vol. 2, 112, 1959.

Forest J., L. Eliasson, A. Hilgers, A New Spacecraft Plasma Simulation Software, PicUp3D/SPIS, in proceedings of 7th Spacecraft Charging Technology Conference, pp.515-520, ESA/SP-476, ISBN No 92-9092-745-3, ESA-ESTEC, Noordwijk, The Netherlands, 23-27 April 2001.

Grard, R. J. L., Properties of the satellite photo-electron sheath derived from photoemission laboratory measurements, J. Geopghys. Res., vol. 78, No16, 2885-2907, 1973.

Guernsey, R.L., and Fu, J.H.M., Potential distribution surrounding a photoemitting plate in a dilute plasma, J. Geophys. Res., 75(16), 3193, 1970.

Hamelin, M., M. Bouhram, N. Dubouloz, M. Malingre, S.A. Grigoriev, and L.V. Zinin, Electrostatic interaction between Interball-2 and the ambient plasma. 2 Influence on the low energy ion measurements with Hyperboloid, Ann. Geophys., 20, 377, 2002.

Hilgers, A. , Interaction between biased sunlit electron collectors in an infinite-Debyelength magnetoplasma: electron temperature threshold effect , J. Geophys. Res., 100, 5705-5713, 1995. 
Laframboise J.G., Theory of spherical and cylindrical Langmuir probes in a collisionless Maxwellian plasma at rest, Institute for aerospace studies, university of Toronto, 1966.

Laframboise J.G. and Stauffer A.D.,Optimum discrete approximation of the Maxwell distribution, AIAA Journal, 7, 3, 520-523, 1969.

Parrot M.J.M. Storey L.R.O. Parker L.W. Laframboise J.G., Theory of cylindrical and spherical Langmuir probes in the limit of vanishing Debye number, Phys. Fluids, Vol. 25, No. 12, 2388-2400, 1982.

Parker, L. W., Plasmasheath-photosheath theory for large high-voltage space structures, in Space systems and their interaction with Earth's space environment, Ed. by H. B. Garrett and C. P. Pike, Vol. 71, Progress in Astronautics and Aeronautics, New York, USA, 1980.

Pedersen, A., C. A. Cattel, C.-G. Faelthammar, V. Formisano, P.-A. Lindqvist, F. Mozer, and R. Torbert, Quasistatic electric field measurements with spherical double probes on the GEOS and ISEE satellites, Space Sci. Rev., 37, pp 269-312, 1984.

Schmidt, R., et al., Results from active spacecraft potential control on the Geotail spacecraft, J. Geophys. Res., 100, 17253,1995.

Scime, E. E., J. Phillips, and S. Bame, Effects of spacecraft potential on three-dimensional electron measurements in the solar wind, J. Geophys. Res., 99, 14,769-14,776, 1994.

Steen N.M., Byrne G.D., Gelbard E.M., Gaussian quadratures for the integrals, Mathematics of comput., vol. 23, pp 661-671, 1969.

Szita, S., A. N. Fazakerley, P. J. Carter, A. M. James, P. Travnicek, G. Watson, M. Andre, A. Eriksson, and K. Torkar, Cluster PEACE observations of electrons of spacecraft origin, Ann. Geophys., 19, 1-10, 2001. 
THIEBAULT ET AL.: ELECTROSTATIC SHEATH AROUND A MAGNETOSPHERIC SPACECRAFT X - 29

Whipple, E. C., Theory of the spherically symmetric photo-electron sheath: a thick sheath approximation and comparison with the ATS 6 observation of a potential barrier, J. Geophys. Res., 81, 601, 1976.

Wrenn G. and Heikkila, Photo-electrons emitted from ISIS Spacecraft, in Photon and particle interactions with surfaces in space, 221-230, 1973.

Zhao H., Schmidt R., Escoubet C.P., Torkar K., Riedler W., Self-consistent determination of the electrostatic potential barrier due to the photoelectron sheath near a spacecraft, Journal of geophysical research, Vol. 101, No. A7, 15,653-15659, 1996. 


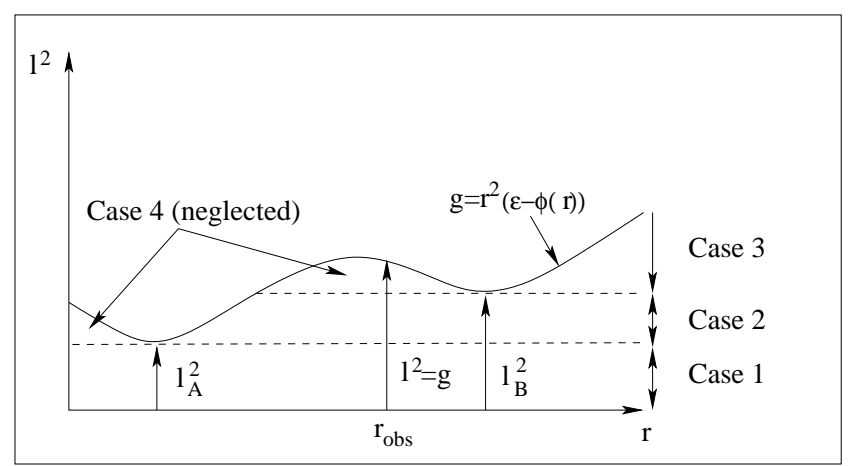

Figure 1. Example of domains of integration in $l_{i}$ for given potential profile and location for attracted particles from the ambient plasma.

Table 1. Parameters for Laframboise's model

\begin{tabular}{l|cc}
\hline Plasma temperature & 1.0 & $\mathrm{eV}$ \\
Plasma number density & 55 & $\mathrm{~cm}^{-3}$ \\
Debye length & 1.0 & $\mathrm{~m}$ \\
Spacecraft potential & 25 & $\mathrm{~V}$ \\
Spacecraft radius & 1.0 & $\mathrm{~m}$ \\
\hline
\end{tabular}




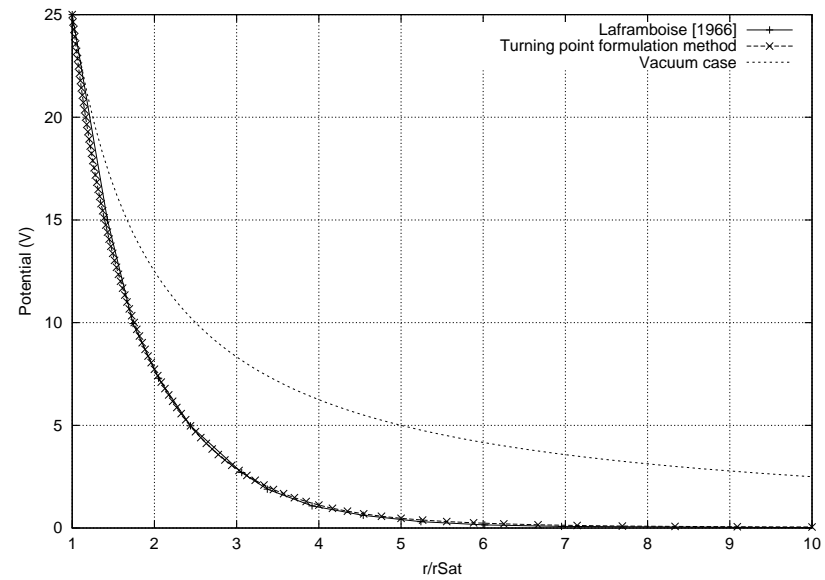

Figure 2. Comparison of the turning point method with results from Laframboise [1966], in the case of a Langmuir probe in a Maxwellian plasma at equilibrium without photoemission. The vacuum solution is also given for information.

Table 2. Parameters for the PicUp simulations

\begin{tabular}{l|lc}
\hline Plasma temperature & 1.0 & $\mathrm{eV}$ \\
Photoelectrons temperature & 2.5 & $\mathrm{eV}$ \\
Photoelectrons current density & 50 & $\mu \mathrm{A} \cdot \mathrm{m}^{-2}$ \\
Plasma number density & 100 & $\mathrm{~cm}^{-3}$ \\
Debye length & 0.74 & $\mathrm{~m}$ \\
Spacecraft potential & $-1,0$, and 1 & $\mathrm{~V}$ \\
Spacecraft radius & 1.0 & $\mathrm{~m}$ \\
\hline
\end{tabular}




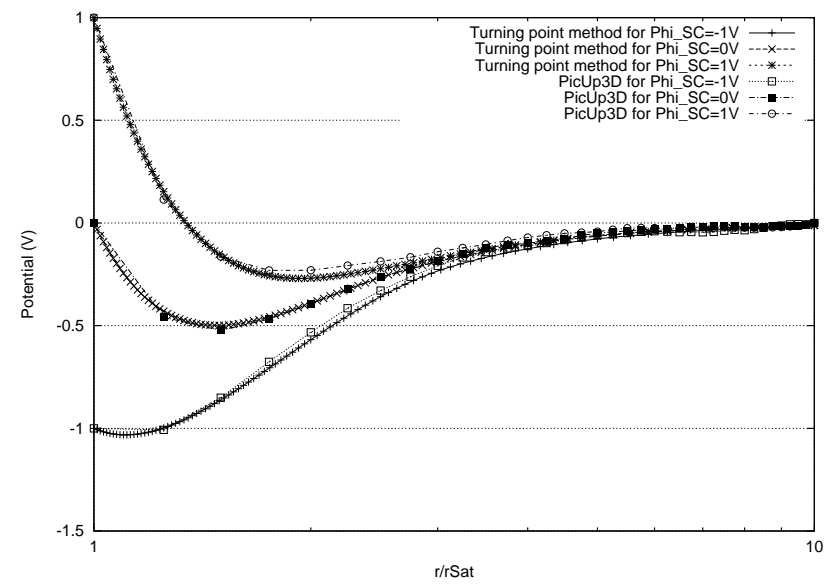

Figure 3. Comparison of the turning point method results with a PIC code in the case of a spherical photoemission.

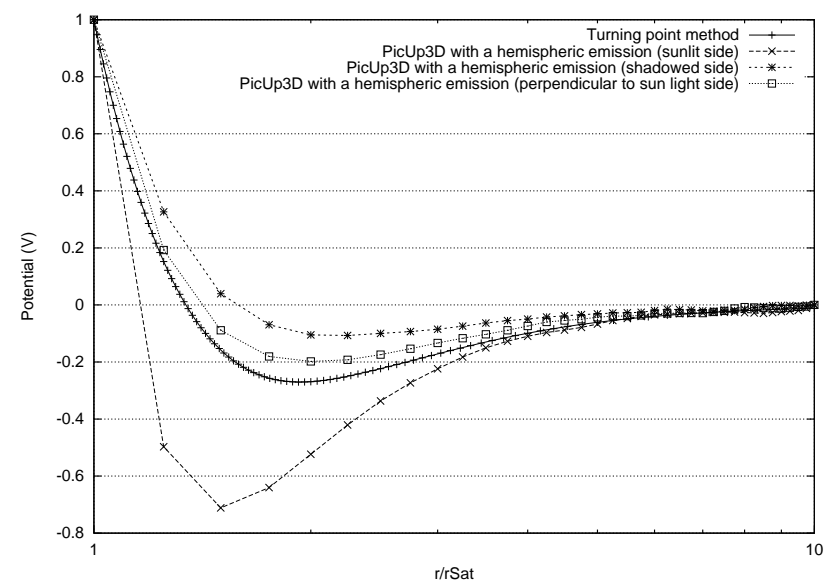

Figure 4. Comparison of the results of the turning point method (in spherical symmetry) and a 3D PIC code with hemispherical photoemission. 
THIEBAULT ET AL.: ELECTROSTATIC SHEATH AROUND A MAGNETOSPHERIC SPACECRAFT X - 33

Table 3. Parameters for the Geotail simulations

\begin{tabular}{l|lc}
\hline Plasma temperature & 100 & $\mathrm{eV}$ \\
Photoelectrons temperature & 1.5 & $\mathrm{eV}$ \\
Plasma number density & 1.0 & $\mathrm{~cm}^{-3}$ \\
Debye length & 74 & $\mathrm{~m}$ \\
Spacecraft potential & 2.6 to 5.4 & $\mathrm{~V}$ \\
Spacecraft radius & 1.1 & $\mathrm{~m}$ \\
Averaged photoelectron current density & 12.5 & $\mu \mathrm{A} . \mathrm{m}^{-2}$ \\
Total photoelectron current & 190 & $\mu \mathrm{A}$ \\
\hline
\end{tabular}

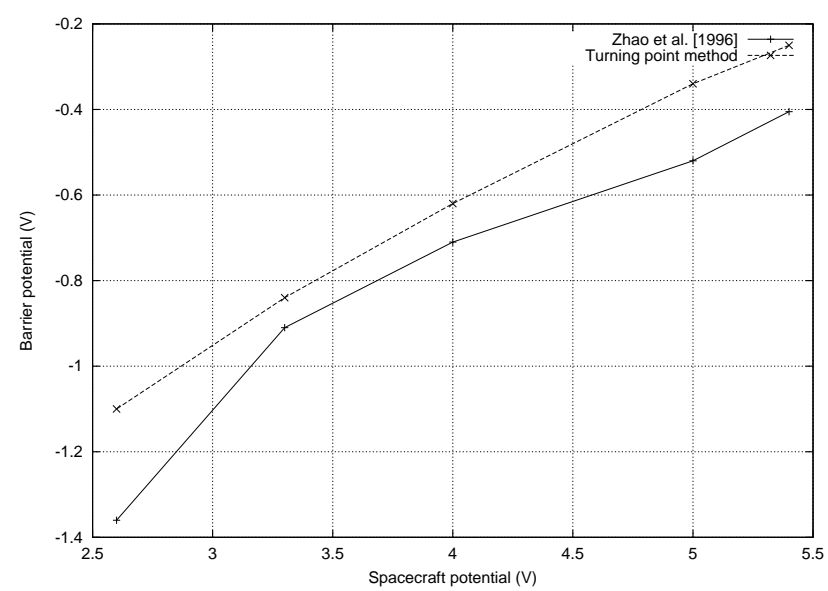

Figure 5. Potential barrier height $\Phi_{B}$ versus the Geotail spacecraft potential as computed by Zhao et al. [1996] (solid line) and by the turning point method (dashed line). 


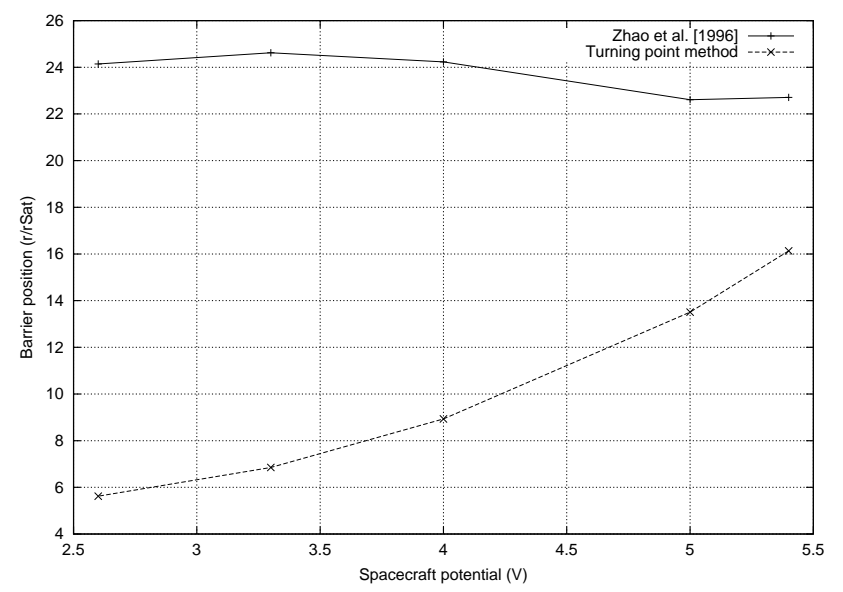

Figure 6. Position of the minimum of the potential barrier versus the Geotail spacecraft potential as computed by Zhao et al. [1996] (solid line) and by the turning point method (dashed line).

Table 4. Parameters for the Cluster simulations of Figures 7, 8, 9 and 10

\begin{tabular}{l|lc}
\hline Plasma temperature & 100 & $\mathrm{eV}$ \\
Plasma number density & 1 & $\mathrm{~cm}^{-3}$ \\
Debye length & 74 & $\mathrm{~m}$ \\
Spacecraft potential & 0.0 to 6.5 & $\mathrm{~V}$ \\
Equivalent sphere radius & 1.41 & $\mathrm{~m}$ \\
Averaged photoelectron current density & 7.5 & $\mu \mathrm{A} . \mathrm{m}^{-2}$ \\
Total photo-electron current & 189 & $\mu \mathrm{A}$ \\
\hline
\end{tabular}




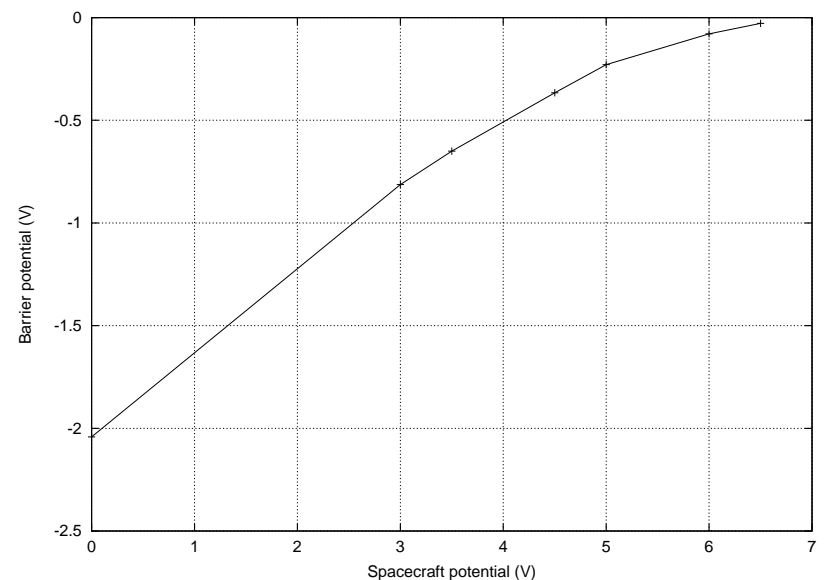

Figure 7. Potential barrier height $\Phi_{B}$ versus spacecraft potential computed with the turning point method for Table 4 simulation parameters.

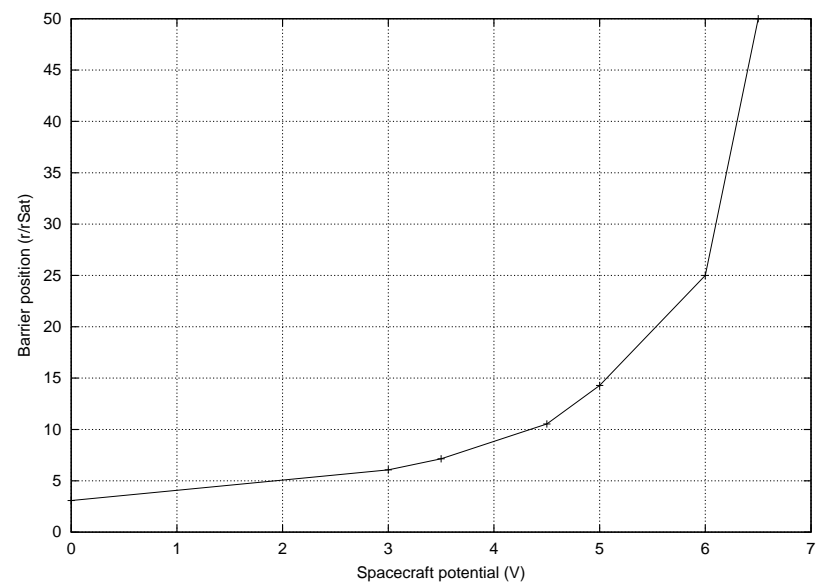

Figure 8. Potential barrier minimum location versus spacecraft potential computed with the turning point method for Table 4 simulation parameters. 


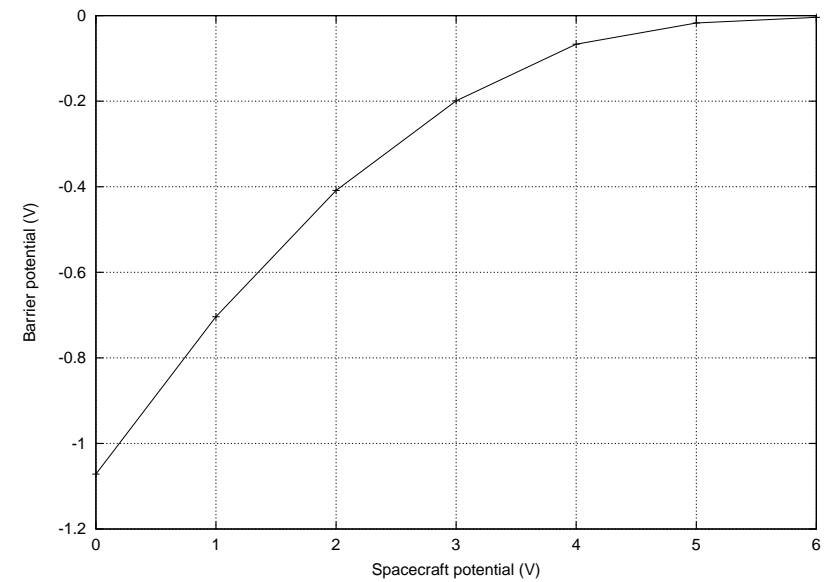

Figure 9. Same as Figure 7 but with a plasma temperature of $1 \mathrm{eV}$.

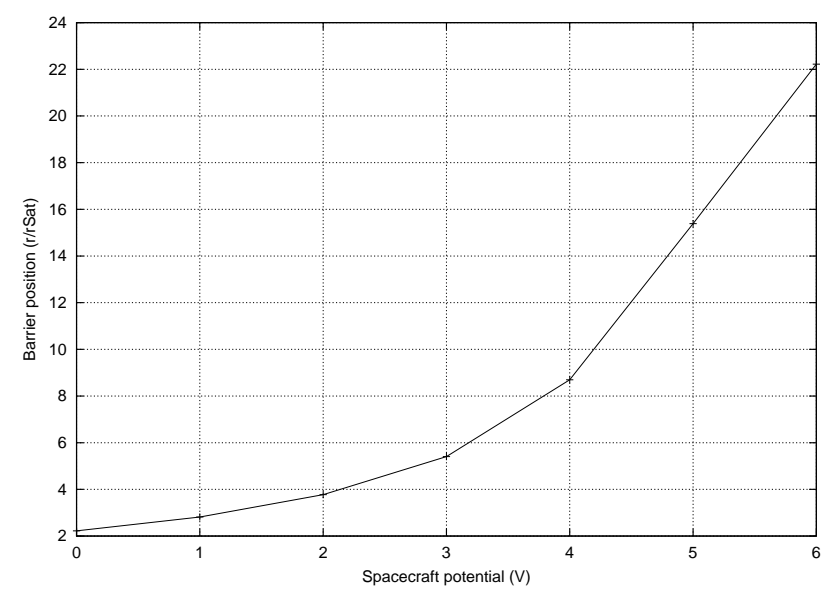

Figure 10. Same as Figure 8 but with a plasma temperature of $1 \mathrm{eV}$. 


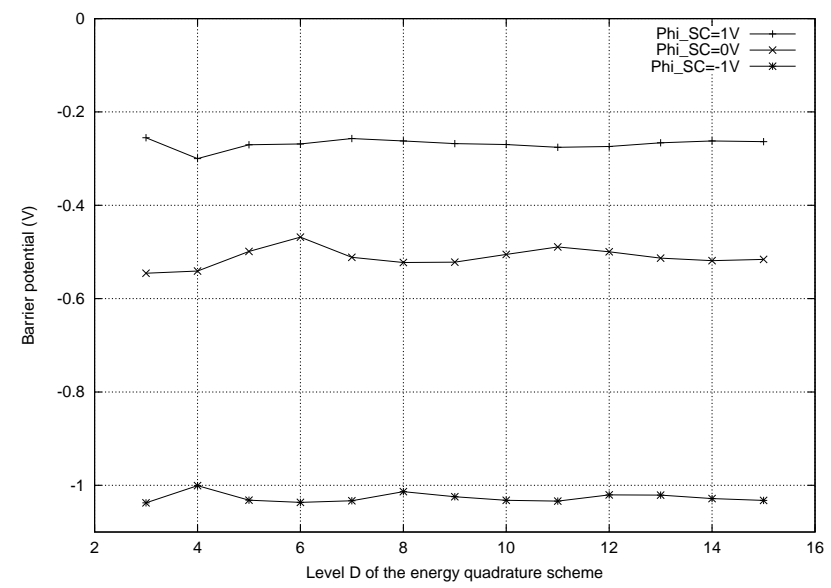

Figure 11. Influence of the energy quadrature degree on the value of the barrier in a case of rapid convergence.

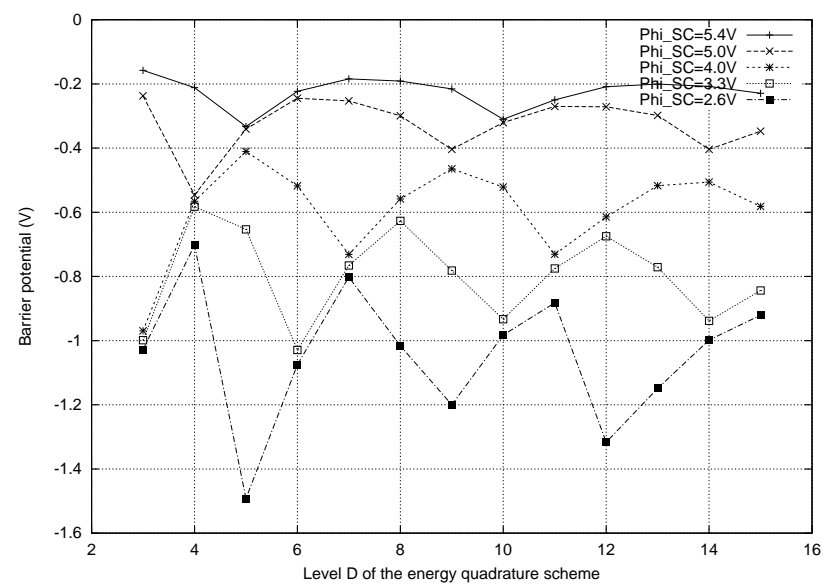

Figure 12. Influence of the energy quadrature uncertainty on the value of the barrier in the case of slow convergence. 
X - 38 ThiebaUlt ET AL.: ELECTROSTATIC SHEATH AROUND A MAGNETOSPHERIC SPACECRAFT

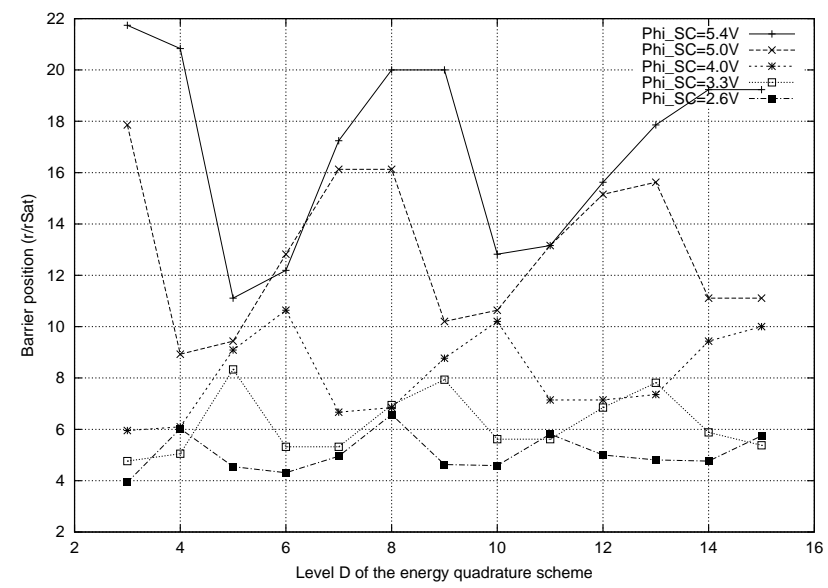

Figure 13. Influence of the energy quadrature degree on the position of the barrier in the case of slow convergence. 